\title{
Inkjet-Printing UHF Antenna for RFID and Sensing Applications on Liquid
} Crystal Polymer

\author{
Amin Rida ${ }^{1}$, Li Yang ${ }^{1}$, Trevale Reynolds ${ }^{1}$, Edward Tan $^{1}$, Symeon Nikolaou ${ }^{2}$ and \\ Manos M. Tentzeris ${ }^{1}$ \\ ${ }^{1}$ School of ECE, Georgia Institute of Technology, Atlanta, GA 30332-0250, USA \\ E-mail: arida@ece.gatech.edu \\ ${ }^{2}$ Frederick University \\ Pallouriotisa, Nicosia 1036, Cyprus \\ s.nikolaou@frederick.ac.cy
}

\begin{abstract}
In this paper, an inkjet-printed broadband antenna on flexible and organic substrate for the UHF Radio Frequency Identification (RFID) and sensing applications is presented. A CPW-fed Monopole configuration is selected due to its many attractive characteristics. The sample is fabricated using inkjet-printing technology on a flexible, low cost Liquid Crystal Polymer (LCP) substrate utilizing a simple, fast, and environmentally friendly process. The design characteristics of the antenna are explained and the simulated results are compared with measurements showing good agreement.
\end{abstract}

\section{Introduction}

The motivation of this work starts with the increasing demand for ubiquitous automatic identification and wireless sensing. The two main factors targeted are cost and performance. While cost is a major key requirement for the mass deployment or RFID and sensor components, performance is equally important especially as technologies are emerging toward higher frequencies as they are bandwidth hungry. That being said, the two main parameters in the system cost analysis for good high frequency performance are the material and process used in fabrication. This paper will stress a low cost, fast, simple, yet efficient process of printing conductive silver nano-particles on one of the lowest cost substrates available in the market [1]. While [1] has explored the printing on ultra low cost paper; it still has limitations as we go up in frequency and yet can face performance issues especially with water absorption and humidity. The next sections of this paper will describe LCP as a low cost high performance microwave substrate, the inkjet printing process, while giving a simple antenna example showing the proof of concept of the merging of inkjet printing and LCP technologies.

\section{LCP Substrate for High Frequency Applications}

The low loss $(\tan \delta=0.002-0.005)$ up to mm-wave frequency range, near hermetic nature (water absorption $<0.04 \%$ ), low-temperature and low-cost-large-format processing on an organic platform, make LCP appealing for high frequency designs where excellent performance is required for minimal cost [2]. LCP's low water absorption makes it stable across a wide range of environments by preventing changes in the relative dielectric constant $\left(\varepsilon_{r}\right)$ and loss tangent $(\tan \delta)$. 
Its Coefficient of Thermal Expansion (CTE) can also be engineered in the x-y plane to match integration with other materials such as $\mathrm{Si}$ or GaAs which gives LCP a broad range of applications. In addition, multilayer circuits are possible with LCP due to two types of LCP material (core layers and bond ply) with different melting temperatures.

\section{Inkjet Printing}

Inkjet-printing is a direct-write technology by which the design pattern is transferred directly to the substrate and does not require masks such as in the conventional etching techniques. Moreover, and unlike etching techniques which are considered subtractive methods since they function by removing unwanted metal from the substrate surface; inkjet-printing jets the single ink droplet from the nozzle to the desired position, therefore, no waste is created, resulting in a "green" and economical fabrication solution [3].

In order to ensure good conductivity for high performance circuits, silver nanoparticle inks are usually selected. After the silver nano-particle droplet is driven through the nozzle, sintering process is found to be necessary to remove excess solvent and to remove material impurities from the depositions [3]. This is also essential to increasing the bond of the deposition with the substrate as well as a virtually continuous metal conductor, providing a good percolation channel for the conduction electrons to flow. The conductivity of the conductive ink varies from $0.4 \sim 2.5 \times 10^{7}$ Siemens $/ \mathrm{m}$ depending on the curing temperature and duration time. Curing temperature of $200^{\circ} \mathrm{C}$ and duration time of two hours is used in the following fabrication to sufficiently cure the nano-paticle ink on LCP.

\section{UHF Antenna Design and Development}

A CPW-fed rectangular monopole antenna was designed and is shown in Fig. 1, with dimensions: coplanar waveguide signal width $w=3.8 \mathrm{~mm}$, gap spacing $g=0.5 \mathrm{~mm}$ and length $L g=30 \mathrm{~mm}$, hence ground length is also $L g=30 \mathrm{~mm}$. The width of the ground planes is chosen to be $W g=35 \mathrm{~mm}$ and a typical value for the height of radiating element from the ground is chosen $h=11 \mathrm{~mm}, \mathrm{Lr}=40 \mathrm{~mm}$ and $W r=50 \mathrm{~mm}$. This module was then inkjet printed using the procedure described above on 50 microns thick LCP substrate. A photograph of the printer is shown in Fig. 1 and which constitutes the whole setup needed for the printing process. Fig. $2 \mathrm{~b}$ shows the fabricated antenna connected to a typical SMA connector for Sparameters measurements using silver epoxy. The Return Loss results are shown in Fig. 3 a for measurements and simulations showing an overall good agreement, while the shift in frequency migh have occurred due to the connectivity and silver epoxy used in the measurement setup and/or surrounding objects in the lab where the Vector Network Analyser is placed. The radiation pattern is shown for both the $\mathrm{x}-\mathrm{y}$ and $\mathrm{y}-\mathrm{z}$ plane with an omnidirectional pattern and a simulated gain of $0.7 \mathrm{~dB}$ at $1 \mathrm{GHz}$. 


\section{Conclusion}

A simple CPW-fed monopole antenna has been designed, developed and characterized on LCP material, a low cost, flexible and environmental friendly microwave substrate; while the inkjet printing, an environmentally friendly fabrication technology, have been successful for the first time. This shows the proof of concept for using ink-jet printing for various applications especially in RFID and sensing.

\section{References:}

[1] A. Rida, L. Yang, and M. M. Tentzeris, "Design and characterization of novel paper-based inkjet-printed UHF antennas for RFID and sensing applications" Procs. of the 2007 IEEEAPS Symposium, pp2749-2752, Honolulu, HI, July 2007

[2] D. Thompson, G. Ponchak, M. M. Tentzeris, J. Papapolymerou, "Characterization of LCP material and transmission lines on LCP substrates from 30 to $110 \mathrm{GHz}$," IEEE Trans. Microwave Theory and Tech., vol. 52, no. 4, pp. 1343-1352, April 2004.

[3] L. Yang, A. Rida, R. Vyas, M. M. Tentzeris, "RFID Tag and RF Structures on a Paper Substrate Using Inkjet-Printing Technology," Microwave Theory and Techniques, IEEE Transactions on Volume 55, Issue 12, Part 2, Dec. 2007 Page(s):2894 - 2901

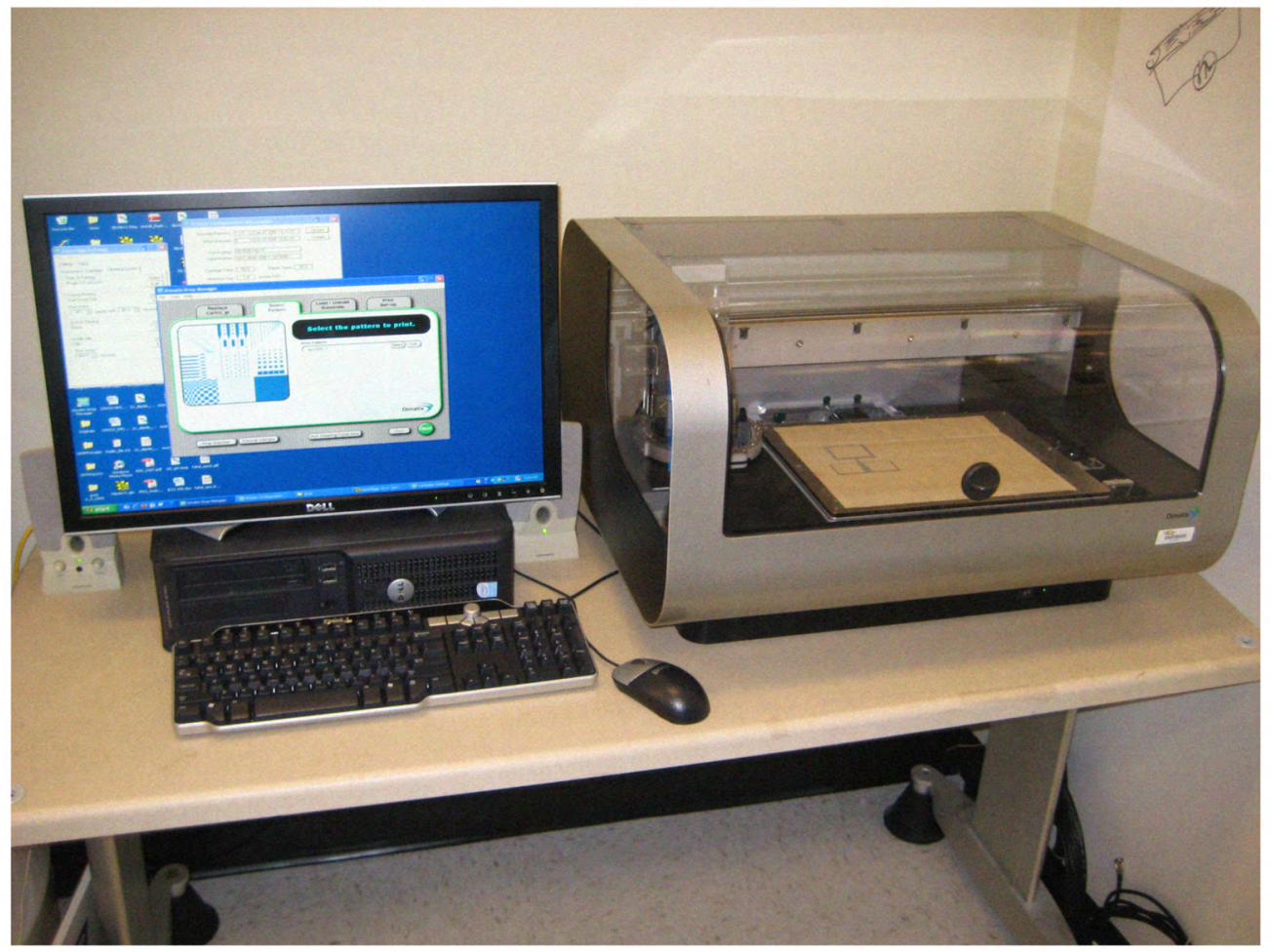

Figure 1. Photograph of Inkjet Printer 


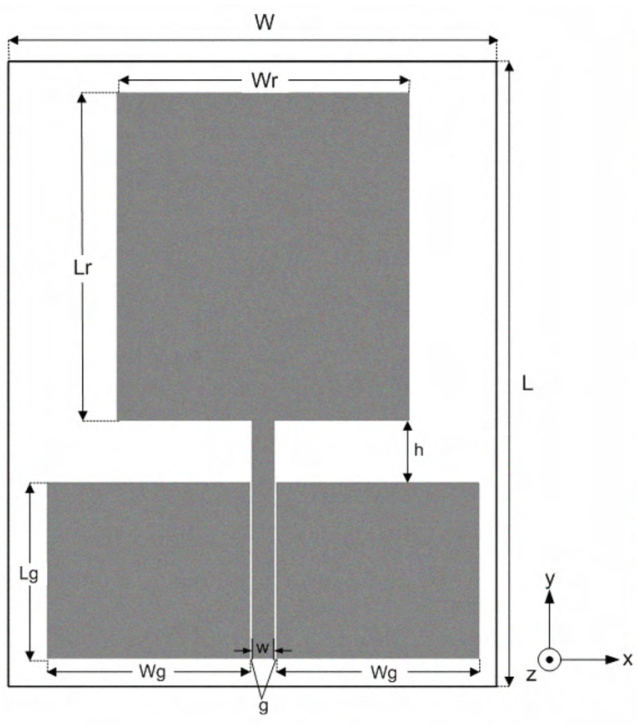

(a)

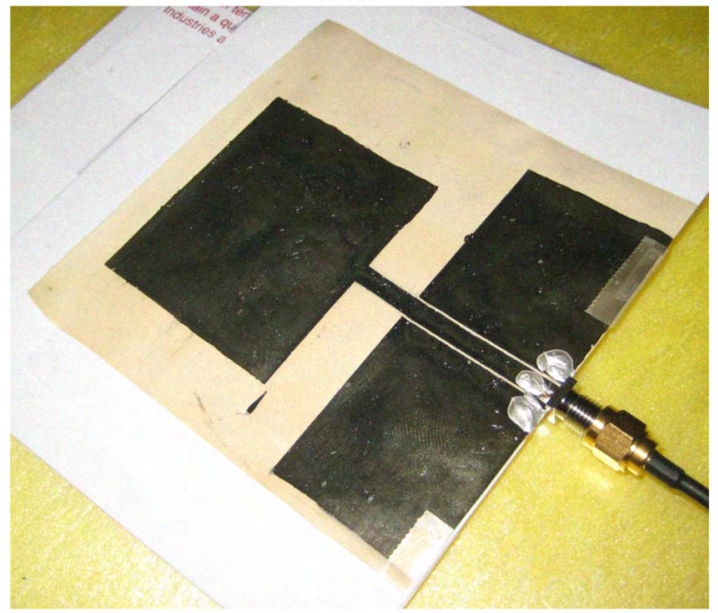

(b)

Figure 2. (a) Planar rectangular UHF monopole antenna configuration and (b)Photograph of fabricated Antenna

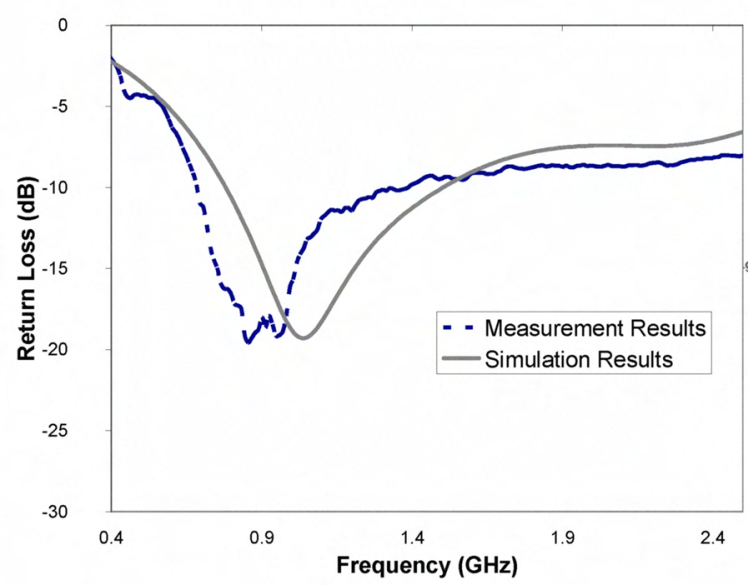

(a)

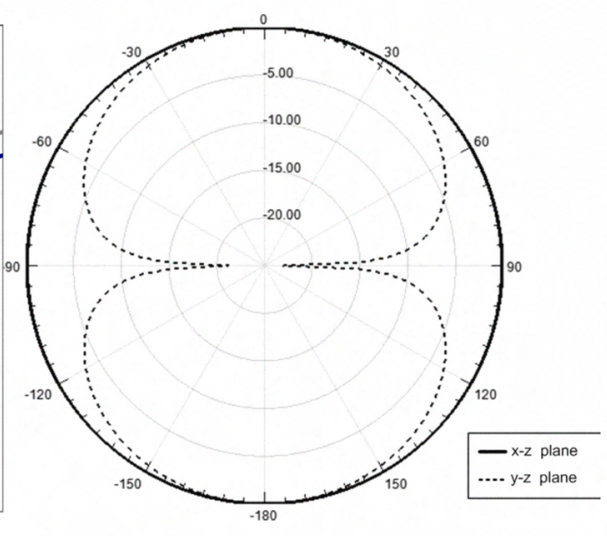

(b)

Figure 3. (a) Measurement and Simulation Results for the Return Loss of the CPW-Monopole Antenna and (b) Omnidirectional radiation pattern of RFID UHF Antenna. 